
\title{
25 Research Square \\ Protective Effects of Ginsenoside Rc Against Acute Cold Exposure Induced Myocardial Injury in Rats
}

\section{Yan Xue}

Jilin University

Xiao-Feng Yu

Jilin University

Xiu-Hang Zhang

Jilin University First Hospital

Ping Yu

Jilin University

Yuan-Geng Li

Jilin University

Wen-Wen Fu

Jilin University

Jia-Ao Yu

Jilin University First Hospital

Da-Yun Sui ( $\nabla$ suidayun@hotmail.com )

Jilin University https://orcid.org/0000-0003-1683-1602

\section{Research}

Keywords: Ginsenoside Rc, Acute cold exposure, Myocardial injury, Inflammation, Apoptosis, SIRT1

Posted Date: June 4th, 2020

DOI: https://doi.org/10.21203/rs.3.rs-32080/v1

License: (c) (1) This work is licensed under a Creative Commons Attribution 4.0 International License. Read Full License

Version of Record: A version of this preprint was published at Journal of Food Science on June 19th, 2021. See the published version at https://doi.org/10.1111/1750-3841.15757. 


\section{Abstract}

Background: Ginsenoside Rc is one of the cardinal bioactive components of Panax ginseng that has been studied for various biological activities. This study aimed to investigate the protective effects of ginsenoside Rc against acute cold exposure induced myocardial injury in rats.

Methods: The rats were intragastrically administrated with ginsenoside Rc $(10,20 \mathrm{mg} / \mathrm{kg})$ or vehicle daily for 7 days. One hour after the seventh-day administration, $3 \%$ pentobarbital sodium was used to anesthetize the rats. Rats of the control group were kept in room temperature $\left(22 \pm 1^{\circ} \mathrm{C}\right)$ and the other groups of rats were exposed to low ambient temperature $\left(-15 \pm 1^{\circ} \mathrm{C}\right)$ in a cold chamber for $6 \mathrm{~h}$. Cardiac function was monitored and recorded as well. To evaluate the protective effects of ginsenoside Rc, we also measured myocardial specific enzymes (lactate dehydrogenase, aspartate aminotransferase and creatine kinase-MB) in plasma, whole blood viscosity (WBV), plasma viscosity (PV), erythrocyte sedimentation rate (ESR) and hematocrit (HCT). The pathological changes of myocardium were observed through histopathological examination. The mRNA expression levels of TNF-a, IL-1 $\beta$ and IL- 6 were by real-time quantitative PCR analysis. And the expressions of silent information regulator1 (SIRT1), B-cell lymphoma-2 (Bcl-2), Bcl-2-associated X (Bax) and Procaspase-3 in heart tissues were measured by Western blot method.

Results: Ginsenoside Rc alone had no effect on cardiac function, myocardial enzyme activities and hemorheology in normal rats. Compared with the model group, pretreatment with ginsenoside Rc significantly improved cardiac function, diminished myocardial specific enzymes release and decreased erythrocyte aggregation index. As a result, the pretreatment regulated abnormal hemorheology, attenuated myocardial histological changes and structural abnormalities, reduced the mRNA expression levels of cardiac inflammatory markers. Ginsenoside Rc also resulted in up-regulating SIRT1, Bcl-2 and Procaspase-3 expressions and down-regulating Bax expression.

Conclusion: The current study suggests that ginsenoside Rc alleviates acute cold exposure induced myocardial injury in rats by inhibiting cardiomyocyte apoptosis via SIRT1 and reducing inflammatory response.

\section{Background}

Exposure to cold weather will have many health effects on human beings, mainly affecting military personnel, homeless people and adventurers (1). In particular, extreme cold temperature condition is a vital risk factor associated with increased cardiovascular morbidity and mortality (2). Many researches have confirmed an association of cold exposure with adverse cardiovascular responses, for example, increased heart rate, blood pressure, systemic vascular resistance and levels of plasma norepinephrine. All of these detrimental reactions can potentially lead to arrhythmias, myocardial ischemia and infarction $(2-4)$. Whereas, the exact mechanisms of these adverse effects in the myocardium under cold exposure remain unclear. 
Panax ginseng C. A. Meyer ( $P$. ginseng) is an herbal plant that has been used as a traditional medicine in Asian countries for thousands of years. Ginsenosides are the principle active constituent found in $P$. ginseng and exhibit anti-oxidative, anti-inflammatory and anti-cancer activities (5-7). Ginsenoside Rc is one of protopanaxadiol type ginsenosides that has been studied for various biological activities (8-10) (Fig. 1). It is reported that ginsenoside Rc can suppress oxidative stress by influencing FoxO1 activity via Akt pathway in human embryo kidney 293T cells (10).

The mammalian sirtuins are a highly conserved family of $\mathrm{NAD}^{+}$-dependent enzymes that regulate cellular stress resistance, energy metabolism, genomic stability and tumorigenesis (11). Silent information regulator 1 (SIRT1) is one of the sirtuins family which is implicated in cell survival under stress and longevity in mammals (12-14). Several studies have suggested that SIRT1 plays a critical role in myocardial injury. SIRT1 has cardioprotective effects in the ischemia-reperfusion injury to cardial muscle (15). Another reseach elucidated that moderate expression of SIRT1 induces resistance to apoptosis and oxidative stress in the heart (16).

It is still not known, however, whether ginsenoside Rc has protective effects in myocardial injury. In the current study, we aimed to evaluate the effective impacts and latent mechanisms of ginsenoside Rc on protecting myocardial injury in acute cold exposure model rats.

\section{Methods}

\section{Animals}

Male Wistar rats (240 to $260 \mathrm{~g}$ ) were from Liaoning Changsheng Biotechnology Co. Ltd. (Benxi, China). Animals were housed and maintained on a 12-h light/dark cycle at a controlled temperature and humidity with unlimited access to food and water. The experiments were approved by the Ethics Committee of Jilin University and were conducted according to the National Institutes of Health Guidelines for the Care and Use of Laboratory Animals (publication 86 - 23, revised in 1986). Every effort was made to minimize discomfort and to reduce the number of animals used.

\section{Reagents}

Ginsenoside Rc (purity > 98\% by HPLC) was provided by Professor YiFa Zhou (School of Life Sciences, Northeast Normal University). Kits for lactate dehydrogenase (LDH), aspartate aminotransferase (AST), creatine kinase-MB (CK-MB) were obtained from Nanjing Jiancheng Bioengineering Institute (Nanjing, China). Hifair ${ }^{\circledR} \mathbb{1}$ st Strand cDNA Synthesis SuperMix and Hieff® qPCR SYBR Green Master Mix (Low Rox Plus) was bought from YEASEN (Shanghai, China). Antibodies against SIRT1, Caspase-3, Bax and $\mathrm{Bcl}-2$ were bought from Affinity Biosciences (OH, USA). Antibodies against $\beta$-actin and HRP Goat AntiRabbit lgG $(\mathrm{H}+\mathrm{L})$ were bought from Beyotime Institute of Biotechnology (Nantong, China). 


\section{Experimental protocol}

Twenty rats were randomly divided into two groups ( $n=10$ in each group): control and ginsenoside Rc at a dose of $20 \mathrm{mg} / \mathrm{kg}$ groups. The rats of control group were intragastrically administrated with $0.5 \% \mathrm{CMC}$ $\mathrm{Na}$ daily for 7 days. The animals in ginsenoside Rc group were intragastrically treated with ginsenoside Rc daily for 7 days.

Forty rats were randomly divided into four groups ( $n=10$ in each group): control group, model group and ginsenoside Rc (10, $20 \mathrm{mg} / \mathrm{kg})$ groups. The rats of control and model groups were intragastrically administrated with $0.5 \% \mathrm{CMC}$-Na daily for 7 days. The animals in ginsenoside Rc groups were intragastrically treated with ginsenoside Rc at doses of 10 or $20 \mathrm{mg} / \mathrm{kg}$, daily for 7 days. One hour after the seventh-day administration, $3 \%$ pentobarbital sodium was used to anesthetize the rats. The rats of control group were kept in room temperature $\left(22 \pm 1^{\circ} \mathrm{C}\right)$ and the other groups of rats were exposed to low ambient temperature $\left(-15 \pm 1^{\circ} \mathrm{C}\right)$ in a cold chamber for $6 \mathrm{~h}(17)$.

\section{Cardiac function evaluation}

Rats were anesthetized and evaluated for cardiac function immediately after acute cold exposure. A $2 \mathrm{~F}$ polyethylene catheter was inserted into the left ventricle by the right common carotid artery. The catheter was connected with a hemodynamic analyzing system (Model RM-6000, Nihon Kohden, Japan). Then, left ventricular end diastolic pressure (LVEDP), left ventricular systolic pressure (LVSP), and positive (+ $\mathrm{dp} / \mathrm{dt}$ ) and negative (-dp/dt) maximal values of the first derivative of left ventricular pressure were evaluated.

\section{Myocardial specific enzyme and hemorheology assays}

Myocardial specific enzymes (LDH, AST and CK-MB) in plasma were examined using a Roche Cobas 8000 automatic biochemical analyzer (Roche Holding Ltd, Basel, Switzerland). Whole blood viscosity (WBV) and plasma viscosity (PV) were measured by LBY-N6 Compact automatic blood rheometer (Beijing Pulisheng Instrument Co., Ltd., Beijing, China). The erythrocyte sedimentation rate (ESR) was recorded by placing $1 \mathrm{~mL}$ whole blood in the accretion tube for $1 \mathrm{~h}$. Then, the pipette was centrifuged at 4,000 rpm for $30 \mathrm{~min}$, and the hematocrit (HCT) was recorded.

\section{Histopathological examination}

For light microscopic evaluation, tissue sections from the left ventricles were fixed in phosphate buffered $10 \%$ formaldehyde buffer at room temperature. The specimens embedded with paraffin were cut into 3$4 \mu \mathrm{m}$ thick sections and stained with hematoxylin-eosin (HE) (18). The sections were examined by an 
experienced observer who was blind to the treatment under light microscope and then photomicrographs were taken.

\section{Real-time quantitative PCR analysis}

The total RNA from cardiac tissue was prepared using TRIzol (Thermo Fisher, USA) following the manufacturer's protocols for RT-qPCR. Synthesis of cDNA and qPCR were determined using Hifair $\mathbb{B} 1 \mathrm{st}$ Strand cDNA Synthesis kit and SYBR Green Master Mix kit (YEASEN, China). The amount of TNF-a, IL-1 $\beta$ and IL- 6 mRNA was normalized to the expression of $\beta$-actin. All of the primers were synthesized by Beijing Dingguo Changsheng Biotechnology Co. Ltd. The following primers were used: TNF-a forward 5'GTCGTAGCAAACCACCAAGC-3', reverse 5'-TGTGGGTGAGGAGCACGTAG-3'; IL-1 $\beta$ forward 5'GCAATGGTCGGGACATAGTT-3', reverse 5'-AGACCTGACTTGGCAGAGG-3'; IL-6 forward 5'AGTGCATCATCGCTGTTCATACA-3', reverse 5'-ATATGTTCTCAGGGAGATCTTGGAA-3'; $\beta$-actin forward 5'GATCAAGATCATTGCTCCTCCTG-3', reverse 5'-AGGGTGTAAAACGCAGCTCA-3'. Fold changes in the target gene expression were calculated by the relative quantification method $\left(2^{-\triangle \Delta C T}\right)$.

\section{Western blot}

The proteins of heart tissue samples were loaded $(50 \mu \mathrm{g})$ and subjected to $10 \%$ or $12 \%$ sodium dodecyl sulfate-polyacrylamide gel electrophoresis and subsequently transferred onto polyvinylidene fluoride membranes (EMD Millipore Corporation, Billerica, USA) for $1.5 \mathrm{~h}$ at $100 \mathrm{~V}$. The membranes were blocked with $5 \%$ nonfat milk for $1 \mathrm{~h}$ and incubated with the primary antibodies: rabbit anti-SIRT1 (1:2,000, Affinity Biosciences, OH, USA), rabbit anti-caspase-3(1:1,000, Affinity Biosciences, OH, USA), rabbit anti-Bax (1:1,000, Affinity Biosciences, OH, USA), rabbit anti-Bcl-2 (1:1,000, Affinity Biosciences, $\mathrm{OH}, \mathrm{USA})$ or rabbit anti- $\beta$-Actin (1:1,500, Beyotime Institute of Biotechnology, Nantong, China) overnight at $4{ }^{\circ} \mathrm{C}$. The membranes were processed with the horseradish peroxidase-labeled secondary antibody (1:5,000, Beyotime Institute of Biotechnology, Nantong, China). The bands were visualized using ECL plus enhanced chemiluminescence kit (Affinity Biosciences, $\mathrm{OH}, \mathrm{USA}$ ). The intensity of protein bands was measured with the NIH Image J software (version 1.62; National Institutes of Health, Bethesda, MD, USA). The ratio of the density of bands of the detected protein to that of $\beta$-actin was used for statistical analysis.

\section{Statistical analysis}

Experiments were implemented in duplicate/triplicate at least thrice. All values are expressed the mean value \pm standard deviation (SD). Analysis was conducted using SPSS 22.0. Statistical analysis among various groups was conducted by one-way analysis of variance (ANOVA) with Tukey's post hoc test. The $P$ less than 0.05 was considered as statistically significant. 


\section{Results}

\section{Effects of ginsenoside Rc on cardiac function, myocardial enzyme activities and hemorheology in normal rats}

Firstly, we evaluated the effect of ginsenoside Rc treatment on normal rats. Under experimental dosages, pretreatment with ginsenoside Rc had no significant effect on the LVSP, LVEDP, $+\mathrm{dp} / \mathrm{dt}$ and $-\mathrm{dp} / \mathrm{dt}(P>$ 0.05 , compared with the control group, Fig. 2). In addition, the activities of LDH, AST and CK-MB were not significantly changed ( $P>0.05$, compared with the control group, Fig. 3 ). Then, we also examined WBV, including 20,60, and $120 \mathrm{~s}^{-1}$ high and low shear rates, PV, ESR and HCT. There were no significant differences among the 2 groups ( $P>0.05$, Fig. 4). These findings indicated that ginsenoside Rc alone had no effect on cardiac function, myocardial enzyme activities and hemorheology in normal rats.

\section{Effects of ginsenoside Rc on cardiac function in acute cold exposure rats}

The cardiac function of rats was evaluated with a hemodynamic analyzing system. The LVEDP of the rats in the model group was significantly increased, as compared with the control group $(P<0.01)$. The LVSP, the $+\mathrm{dp} / \mathrm{dt}$, and the $-\mathrm{dp} / \mathrm{dt}$ in the model group were markedly decreased $(P<0.01)$. Compared with the model group, LVEDP of the rats in the ginsenoside Rc groups was significantly decreased, whereas the LVSP, the $+\mathrm{dp} / \mathrm{dt}$, and the $-\mathrm{dp} / \mathrm{dt}$ of the ginsenoside Rc groups were increased $(P<0.05$ or $P<0.01)$. These results demonstrated that ginsenoside Rc has a property improving cardiac function in acute cold exposure rats (Fig. 5).

\section{Effects of ginsenoside Rc on myocardial enzyme activities in acute cold exposure rats}

The elevations of cardiac markers enzymes (such as LDH, AST and CK-MB) are important bases for the diagnosis of myocardial injury. As shown in Fig. 6, acute cold exposure resulted in a significant increase in the activities of LDH, AST and CK-MB ( $P<0.01$, compared with the control group). However, pretreatment with ginsenoside Rc (10 and $20 \mathrm{mg} / \mathrm{kg})$ remarkably alleviated these conditions $(P<0.01$, compared with the model group).

\section{Effects of ginsenoside Rc on hemorheology in acute cold exposure rats}

In this study, the results of WBV, including 20,60, and $120 \mathrm{~s}^{-1}$ high and low shear rates, PV, ESR and HCT are shown in Fig. 7. Compared with the control group, WBV, PV, ESR, and HCT in the model group were 
significantly increased $(P<0.01)$. Compared with the model group, pretreatment with ginsenoside Rc (10 and $20 \mathrm{mg} / \mathrm{kg}$ ) notably reduced WBV at high and low shear rates $\left(120,60\right.$ and $\left.20 \mathrm{~s}^{-1}\right), \mathrm{PV}, \mathrm{ESR}$ and HCT $(P<0.05$ or $P<0.01)$. These results indicated that ginsenoside Rc could improve acute cold exposure rats by decreasing erythrocyte aggregation index and regulating abnormal hemorheology.

\section{Effects of ginsenoside Rc on histopathological examination of cardiac tissues in acute cold exposure rats}

As shown in Fig. 8, HE staining of heart tissues in the model group showed myocardial histological changes and structural abnormalities in acute cold exposure rats, including myocardial cell loss, widespread myocardial structure disorder, myocardium fragment and a high number of inflammatory cell infiltration. Pretreatment with ginsenoside Rc (10 and $20 \mathrm{mg} / \mathrm{kg}$ ) significantly attenuated the pathophysiological changes in the cardiac muscle fiber.

\section{Effects of ginsenoside Rc on inflammation response in acute cold exposure rats}

Acute cold exposure induced large amounts of inflammatory factors in myocardium, and then aggravated myocardial damage. The pro-inflammatory factor levels were elevated noticeably in the model group ( $P<0.01$, compared with the control group, Fig. 9). Pretreatment with ginsenoside Rc decreased in the mRNA expression levels of TNF-a, IL-1 $\beta$ and IL-6 $(P<0.01$, compared with the model group, Fig. 9). These results showed that ginsenoside Rc reduced inflammation response in acute cold exposure induced injury.

\section{Effects of ginsenoside Rc on expression of SIRT1, Bcl-2, Bax and Procaspase- 3 in acute cold exposure rats}

We further evaluated the expression of SIRT1 to explore the molecular mechanisms underlying the protective effects of ginsenoside Rc. We found that SIRT1 expression was markedly decreased in the model group $(P<0.01$, compared with the control group, Fig. 10). However, pretreatment of the rats with ginsenoside Rc increased SIRT1 expression ( $P<0.01$, compared with the model group, Fig. 10). We next evaluated the apoptotic-related protein expressions. As shown in Fig. 10, acute cold exposure notably decreased the expression of procaspase 3 and $\mathrm{Bcl}-2$, but increased the expression of $\mathrm{Bax}(P<0.01$, compared with the control group, Fig. 10). In contrast, pretreatment with ginsenoside Rc (10 and $20 \mathrm{mg} / \mathrm{kg})$ decreased the expression of Bax and increased the expression of procaspase-3 and Bcl-2 $(P<$ 0.05 or $P<0.01$, compared with the model group). These results indicated that pretreatment with ginsenoside Rc may activate the SIRT1 expression, thus inhibiting the apoptotic signaling pathway. 


\section{Discussion}

The results show that ginsenoside Rc protects the heart from inflammation and apoptosis. Stimulating endogenous SIRT1 appears to be beneficial to reduce the level of cold exposure induced myocardial injury.

One of mechanisms for cold exposure induced myocardial injury is ischemic. Microcirculatory perfusion dependends not only on vascular contraction and perfusion pressure, but also on rheologic characteristics of the blood. Cold exposure can directly cause cardiovascular stress due to vasoconstriction and variations in blood pressure (19). The effect of hypothermia includes reductions in leukocyte mobility, elevations in plasma viscosity and red cell deformability which may lead to a reduction of blood flow through capillaries (20-22). It further slows oxygen delivery and reduces the elimination of toxic metabolites. Therefore, hypothermia will lead to myocardial dyfunction in clinic (23). However, there are few experiments on cold exposure induced heart injury in rats, and our results can play a complementary role. According to our experiments, ginsenoside Rc can effectively reduce the damage of acute cold exposure to myocardial function in rats.

Cytokines are known as signaling molecules to regulate cellular function and specifically involved in inflammation and immune response as well as in heart disease. Acute cardiac injury is associated with increased tissue level of IL-1 $\beta$ (24). Additionally, IL- 6 also plays a negative role in cardiac injury in most experimental and clinical studies $(25,26)$. Another class of cytokines, the tumor necrosis factor $(T N F)$, is a ubiquitous cell signaling protein produced by various cells. Sustained inflammatory signaling by cardiac overexpression of TNF-a leads to proteotoxicity and cell death in the heart (27). In the present study, the high expression of IL-1 $\beta$, IL- 6 and TNF- $\alpha$ in the myocardium may aggravate myocardial damage. The pretreatment with ginsenoside Rc exhibits anti-inflammatory effect by inhibiting expression of these cytokines.

Our further study shows that acute cold exposure induced cardiomyocyte apoptosis. Previously, several studies have demonstrated that apoptotic death of cardiomyocytes appears after exposure various damaging stimuli both in vitro and in vivo (28-31). SIRT1, Bcl-2 family and caspases are associated with apoptotic cell death in cardiomyocytes (32-34). Bcl-2 is a key mediator in the regulation of cardiac myocyte apoptosis which has a protective effect on mitochondria permeability (35). By contrast, Bcl-2associated X (Bax), a proapoptotic member of the Bcl-2 family, can neutralize the activation of $\mathrm{Bcl}-2$ by forming Bcl-2/Bax heterodimers (36). Bax also directly activates caspase-3 which results in cleavage of cytoskeletal and nuclear proteins (37). SIRT1 was observed in regulating expression of anti-/proapoptotic molecules. It was reported that SIRT1 upregulates Bcl-2 and downregulates Bax during ischemia-reperfusion in SIRT1 transgenic mice (38). Thus, SIRT1 is a potential target in the therapy of cardiac injury. In different models of cardiac injury, resveratrol as a SIRT1 activator has a protective effect $(39,40)$. Our previous study has showed ginsenoside Rg2 alleviates ischemia-reperfusion injury by activating SIRT1 signaling (41). Currently, our results show that ginsenoside Rc inhibits expression of Bax in cardiomyocytes after acute cold exposure. The levels of SIRT1, Bcl-2 and procaspase-3 are upregulated 
by pretreatment of ginsenoside Rc. Thus, our results suggest that ginsenoside Rc inhibits cardiomyocyte apoptosis by activating SIRT1 in rats.

\section{Conclusions}

The current study suggests that ginsenoside Rc alleviates acute cold exposure induced myocardial injury in rats. The mechanisms of ginsenoside Rc involve suppression of inflammatory cytokine production and inhibition of cardiomyocyte apoptosis by activating SIRT1. Our findings indicate that ginsenoside Rc is a key cardioprotective component of $P$. ginseng which may be potentially valuable as a monomeric drug. However, acute cold exposure clinically is mostly unpredictable. The effect of ginsenoside Rc after the occurrence of cold exposure needs to be further studied. Furthermore, the cellular targets of SIRT1 during cardiac injury should be elucidated. Therefore, it is indeed necessary to develop more effective methods to protect the heart from acute cold exposure.

\section{Abbreviations}

ANOVA: analysis of variance; AST: aspartate aminotransferase; Bax: Bcl-2-associated X; Bcl-2: B-cell lymphoma-2; CK-MB: creatine kinase-MB; $+\mathrm{dp} / \mathrm{dt}$ : positive maximal values of the first derivative of left ventricular pressure; -dp/dt: negative maximal values of the first derivative of left ventricular pressure; ESR: erythrocyte sedimentation rate; HCT: hematocrit; HE: hematoxylin-eosin; IL-1 $\beta$ : interleukin-1 $\beta$; IL6:interleukin-6; LDH: lactate dehydrogenase; LVEDP: left ventricular end diastolic pressure; LVSP: left ventricular systolic pressure; NAD+: nicotinamide adenine dinucleotide; $P$. ginseng: Panax ginseng $C$. $A$. Meyer, PV: plasma viscosity; SD: standard deviation; SIRT1: silent information regulator 1; TNF: tumor necrosis factor; TNF-a: tumor necrosis factor-a; WBV: whole blood viscosity.

\section{Declarations}

\section{Ethics approval and consent to participate}

The study was approved by the Ethics Committee of Jilin University. All animals were treated in accordance with the Animal Care and Use Committee of Jilin University (Changchun, China).

\section{Consent for publication}

Not applicable.

\section{Availability of data and materials}

Available from the corresponding author on reasonable request. 


\section{Competing interests}

The authors declare that they have no competing interests.

\section{Funding}

The project design, materials, data interpretation, and writing are supported by the National Natural Science Foundation of China (No. 81473378) and the Natural Science Foundation of Jilin Province, China (No. 20170101002JC).

\section{Authors' contributions}

SDY, YJA and FWW conceived and designed the study. XY, YXF, YP, LYG and FWW performed the experiments. $\mathrm{XY}$ wrote the paper. $\mathrm{XY}, \mathrm{FWW}$ and $\mathrm{ZXH}$ were responsible critical revision of the manuscript. All authors read and approved the final version manuscript for publication.

\section{Acknowledgements}

Not applicable.

\section{References}

1. Rathjen NA, Shahbodaghi SD, Brown JA. Hypothermia and Cold Weather Injuries. Am Fam Physician. 2019;100(11):680-6.

2. Aklilu D, Wang T, Amsalu E, Feng W, Li Z, Li X, et al. Short-term effects of extreme temperatures on cause specific cardiovascular admissions in Beijing, China. Environ Res. 2020;186:109455.

3. Cheng X, Su H. Effects of climatic temperature stress on cardiovascular diseases. Eur J Intern Med. 2010;21(3):164-7.

4. Cold exposure and winter mortality from ischaemic heart disease, cerebrovascular disease, respiratory disease, and all causes in warm and cold regions of Europe. The Eurowinter Group. Lancet. 1997;349(9062):1341-6.

5. Lu JM, Yao Q, Chen C. Ginseng compounds: an update on their molecular mechanisms and medical applications. Curr Vasc Pharmacol. 2009;7(3):293-302.

6. Hofseth LJ, Wargovich MJ. Inflammation, cancer, and targets of ginseng. J Nutr. 2007;137(1 Suppl):183S-5S.

7. Attele AS, Wu JA, Yuan CS. Ginseng pharmacology: multiple constituents and multiple actions. Biochem Pharmacol. 1999;58(11):1685-93. 
8. Jung SY, Choi S, Ko YS, Park CS, Oh S, Koh SR, et al. Effects of ginsenosides on vanilloid receptor (VR1) channels expressed in Xenopus oocytes. Mol Cells. 2001;12(3):342-6.

9. Choi SS, Han EJ, Han KJ, Lee HK, Suh HW. Antinociceptive effects of ginsenosides injected intracerebroventricularly or intrathecally in substance P-induced pain model. Planta Med. 2003;69(11):1001-4.

10. Kim DH, Park CH, Park D, Choi YJ, Park MH, Chung KW, et al. Ginsenoside Rc modulates Akt/FoxO1 pathways and suppresses oxidative stress. Arch Pharm Res. 2014;37(6):813-20.

11. Finkel T, Deng CX, Mostoslavsky R. Recent progress in the biology and physiology of sirtuins. Nature. 2009;460(7255):587-91.

12. Vaziri H, Dessain SK, Ng Eaton E, Imai SI, Frye RA, Pandita TK, et al. hSIR2(SIRT1) functions as an NAD-dependent p53 deacetylase. Cell. 2001;107(2):149-59.

13. Satoh A, Brace CS, Rensing N, Cliften P, Wozniak DF, Herzog ED, et al. SIRT1 extends life span and delays aging in mice through the regulation of Nk2 homeobox 1 in the DMH and LH. Cell Metab. 2013;18(3):416-30.

14. Radak Z, Koltai E, Taylor AW, Higuchi M, Kumagai S, Ohno H, et al. Redox-regulating sirtuins in aging, caloric restriction, and exercise. Free Radic Biol Med. 2013;58:87-97.

15. Yamamoto T, Sadoshima J. Protection of the heart against ischemia/reperfusion by silent information regulator 1. Trends Cardiovasc Med. 2011;21(1):27-32.

16. Alcendor RR, Gao S, Zhai P, Zablocki D, Holle E, Yu X, et al. SIRT1 regulates aging and resistance to oxidative stress in the heart. Circ Res. 2007;100(10):1512-21.

17. Jin-Long M, Gang Z, Xue-feng S, Bin ZW-, Ji-Ye W, Jun CX, et al. Cold stress-induced impairment of learning and memory of rats and its mechanism. Progress in Modern Biomedicine. 2009;9(24):46157.

18. Fu WW, Yu XF, Lu ZY, Sun FF, Wang YC, Zhang Y, et al. Protective effects of ginsenoside Rb2 on myocardial ischemia in vivo and in vitro. Int J Clin Exp Med. 2016;9(6):9843-55.

19. Keatinge WR, Donaldson GC. Cardiovascular mortality in winter. Arctic Med Res. 1995;54 Suppl 2:168.

20. Evans E, Yeung A. Apparent viscosity and cortical tension of blood granulocytes determined by micropipet aspiration. Biophys J. 1989;56(1):151-60.

21. Poulos ND, Mollitt DL. The nature and reversibility of hypothermia-induced alterations of blood viscosity. J Trauma. 1991;31(7):996-8; discussion 8-1000.

22. Lecklin T, Egginton S, Nash GB. Effect of temperature on the resistance of individual red blood cells to flow through capillary-sized apertures. Pflugers Arch. 1996;432(5):753-9.

23. Aslam AF, Aslam AK, Vasavada BC, Khan IA. Hypothermia: evaluation, electrocardiographic manifestations, and management. Am J Med. 2006;119(4):297-301.

24. Pomerantz BJ, Reznikov LL, Harken AH, Dinarello CA. Inhibition of caspase 1 reduces human myocardial ischemic dysfunction via inhibition of IL-18 and IL-1beta. Proc Natl Acad Sci U S A. 
2001;98(5):2871-6.

25. Gabriel AS, Martinsson A, Wretlind B, Ahnve S. IL-6 levels in acute and post myocardial infarction: their relation to CRP levels, infarction size, left ventricular systolic function, and heart failure. Eur $\mathrm{J}$ Intern Med. 2004;15(8):523-8.

26. Dawn B, Xuan YT, Guo Y, Rezazadeh A, Stein AB, Hunt G, et al. IL-6 plays an obligatory role in late preconditioning via JAK-STAT signaling and upregulation of iNOS and COX-2. Cardiovasc Res. 2004;64(1):61-71.

27. Hartupee J, Szalai GD, Wang W, Ma X, Diwan A, Mann DL. Impaired Protein Quality Control During Left Ventricular Remodeling in Mice With Cardiac Restricted Overexpression of Tumor Necrosis Factor. Circ Heart Fail. 2017;10(12).

28. Cheng W, Kajstura J, Nitahara JA, Li B, Reiss K, Liu Y, et al. Programmed myocyte cell death affects the viable myocardium after infarction in rats. Exp Cell Res. 1996;226(2):316-27.

29. Gottlieb RA, Burleson KO, Kloner RA, Babior BM, Engler RL. Reperfusion injury induces apoptosis in rabbit cardiomyocytes. J Clin Invest. 1994;94(4):1621-8.

30. Narula J, Haider N, Virmani R, DiSalvo TG, Kolodgie FD, Hajjar RJ, et al. Apoptosis in myocytes in end-stage heart failure. N Engl J Med. 1996;335(16):1182-9.

31. Umansky SR, Cuenco GM, Khutzian SS, Barr PJ, Tomei LD. Post-ischemic apoptotic death of rat neonatal cardiomyocytes. Cell Death Differ. 1995;2(4):235-41.

32. Bonavita F, Stefanelli C, Giordano E, Columbaro M, Facchini A, Bonafe F, et al. H9c2 cardiac myoblasts undergo apoptosis in a model of ischemia consisting of serum deprivation and hypoxia: inhibition by PMA. FEBS Lett. 2003;536(1-3):85-91.

33. Kirshenbaum LA, de Moissac $D$. The bcl-2 gene product prevents programmed cell death of ventricular myocytes. Circulation. 1997;96(5):1580-5.

34. Yamamura T, Otani $H$, Nakao $Y$, Hattori R, Osako M, Imamura H. IGF-I differentially regulates Bcl-xL and Bax and confers myocardial protection in the rat heart. Am J Physiol Heart Circ Physiol. 2001;280(3):H1191-200.

35. Cook SA, Sugden PH, Clerk A. Regulation of bcl-2 family proteins during development and in response to oxidative stress in cardiac myocytes: association with changes in mitochondrial membrane potential. Circ Res. 1999;85(10):940-9.

36. Wang L, Ma W, Markovich R, Lee WL, Wang PH. Insulin-like growth factor I modulates induction of apoptotic signaling in H9C2 cardiac muscle cells. Endocrinology. 1998;139(3):1354-60.

37. Reed JC. Regulation of apoptosis by bcl-2 family proteins and its role in cancer and chemoresistance. Curr Opin Oncol. 1995;7(6):541-6.

38. Hsu CP, Zhai P, Yamamoto T, Maejima Y, Matsushima S, Hariharan N, et al. Silent information regulator 1 protects the heart from ischemia/reperfusion. Circulation. 2010;122(21):2170-82.

39. Chan AY, Dolinsky VW, Soltys CL, Viollet B, Baksh S, Light PE, et al. Resveratrol inhibits cardiac hypertrophy via AMP-activated protein kinase and Akt. J Biol Chem. 2008;283(35):24194-201. 
40. Gurusamy N, Lekli I, Mukherjee S, Ray D, Ahsan MK, Gherghiceanu M, et al. Cardioprotection by resveratrol: a novel mechanism via autophagy involving the mTORC2 pathway. Cardiovasc Res. 2010;86(1):103-12.

41. Fu WW, Xu HL, Yu XF, Lyu C, Tian Y, Guo MY, et al. 20(S)-Ginsenoside Rg2 attenuates myocardial ischemia/reperfusion injury by reducing oxidative stress and inflammation: role of SIRT1. Rsc Adv. 2018;8(42):23947-62.

Figures

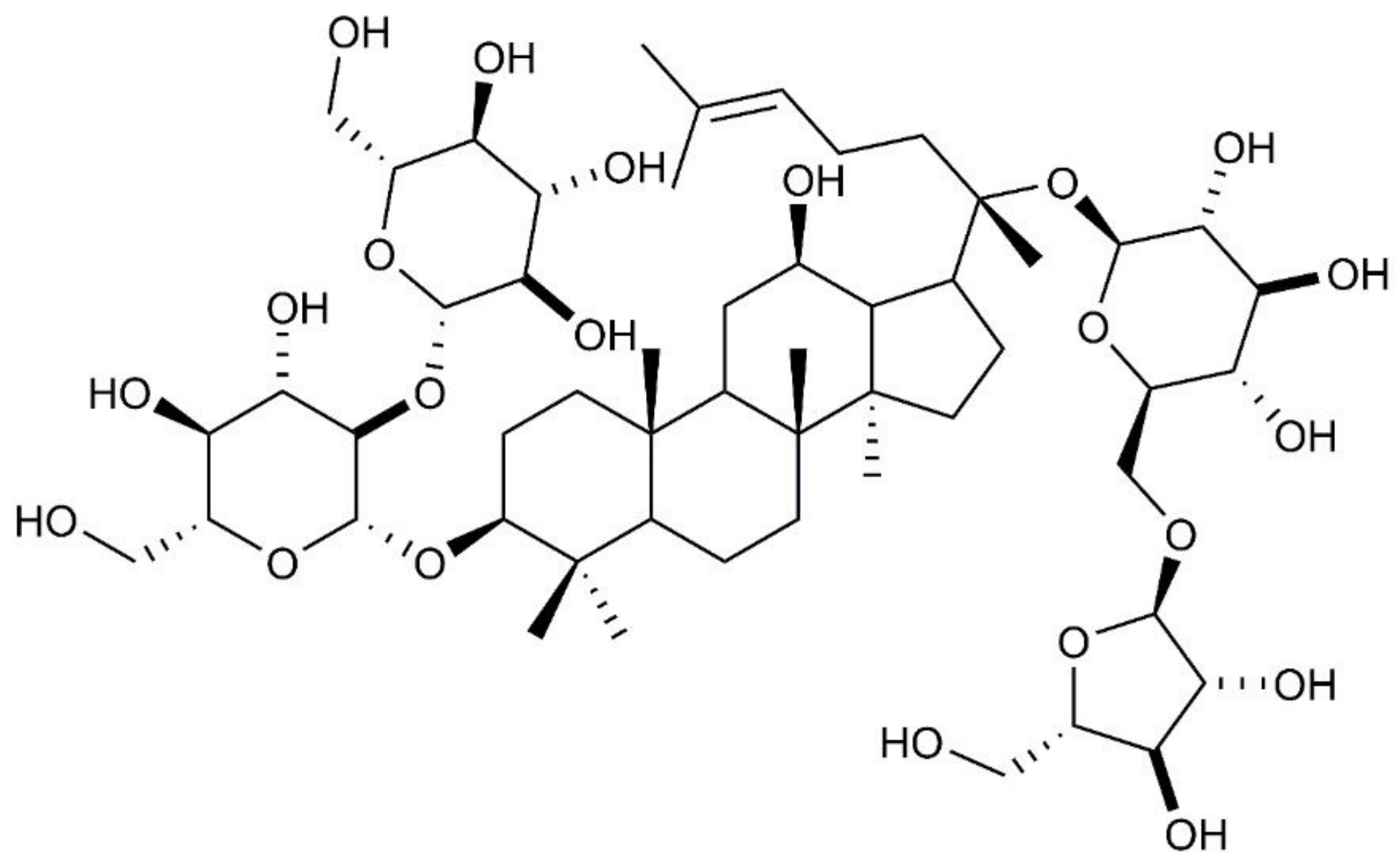

Figure 1

Molecular structure of ginsenoside Rc. 

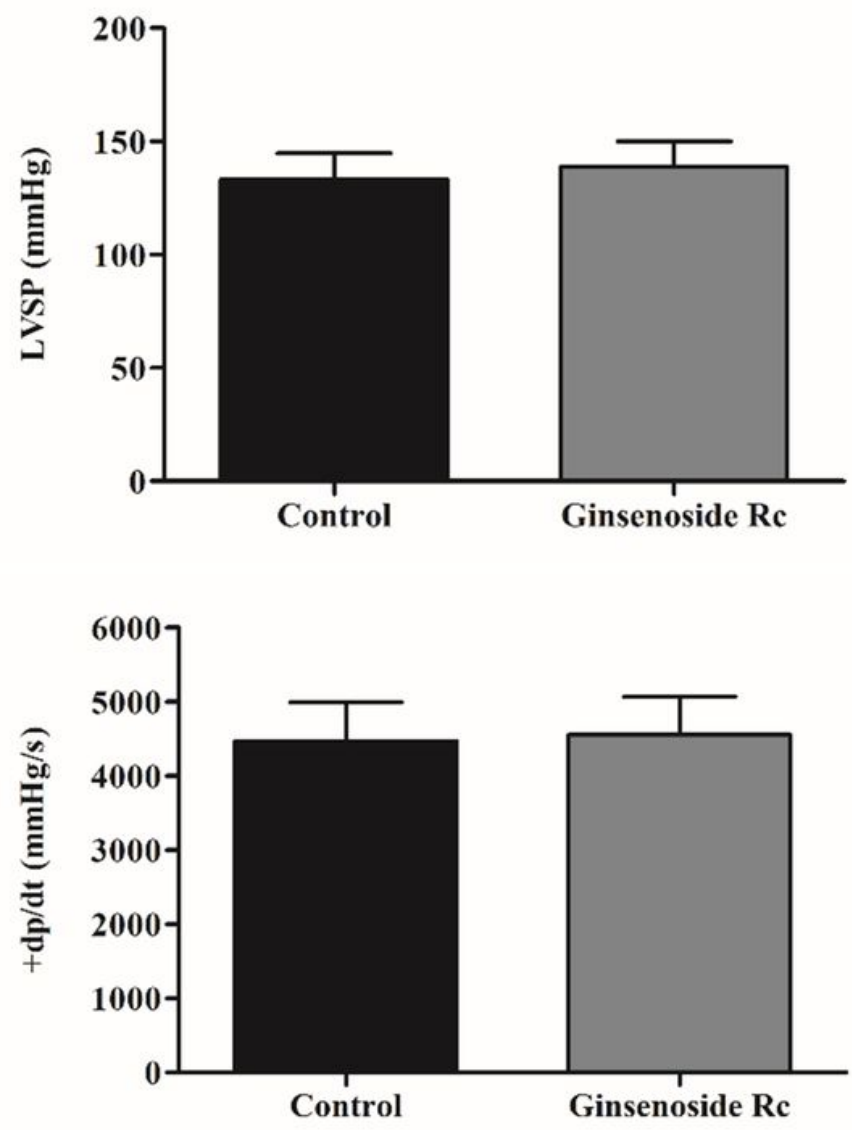
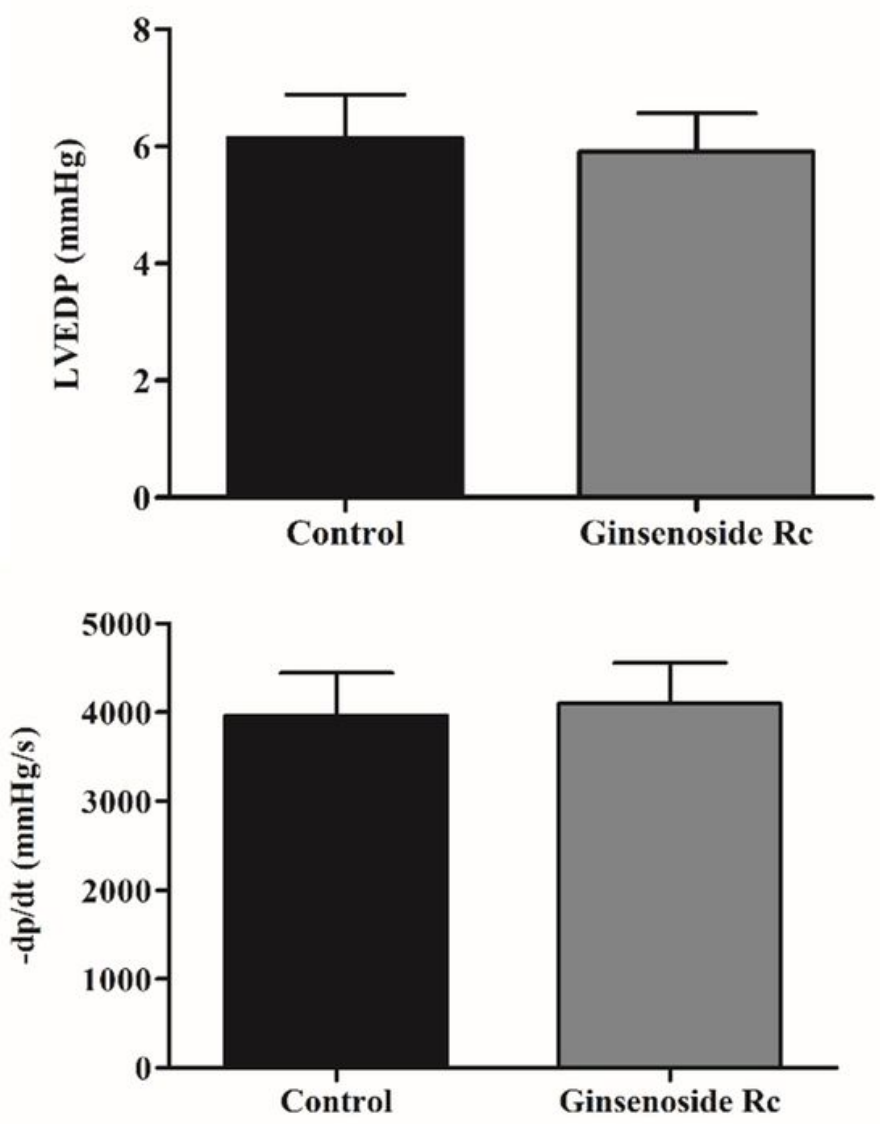

\section{Figure 2}

Effects of ginsenoside Rc on cardiac function in normal rats. Data were presented as the mean \pm SD. $\mathrm{n}=10$ per group. Statistical analysis among various groups was conducted by one-way analysis of variance (ANOVA) with Tukey's post hoc test.
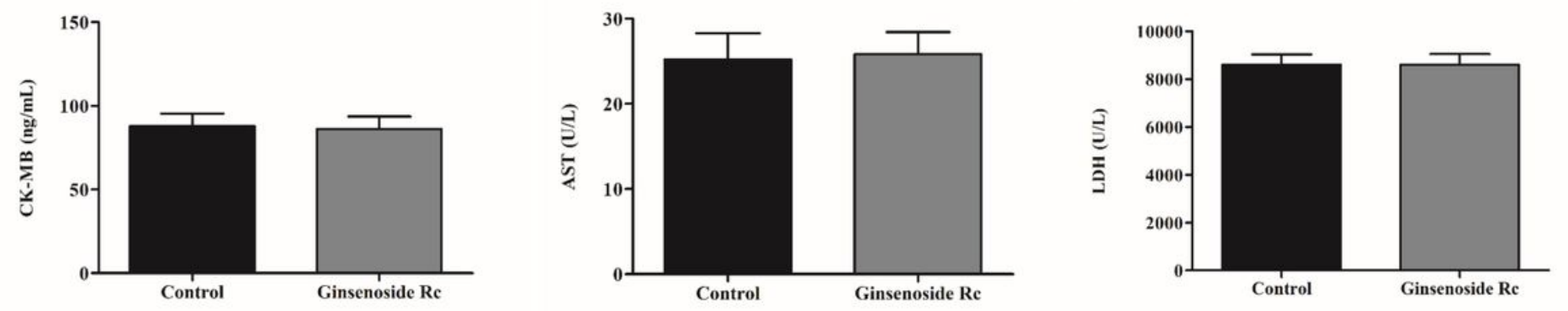

\section{Figure 3}

Effects of ginsenoside Rc on myocardial enzyme activities in normal rats. Data were presented as the mean $\pm S D$. $n=10$ per group. Statistical analysis among various groups was conducted by one-way analysis of variance (ANOVA) with Tukey's post hoc test. 

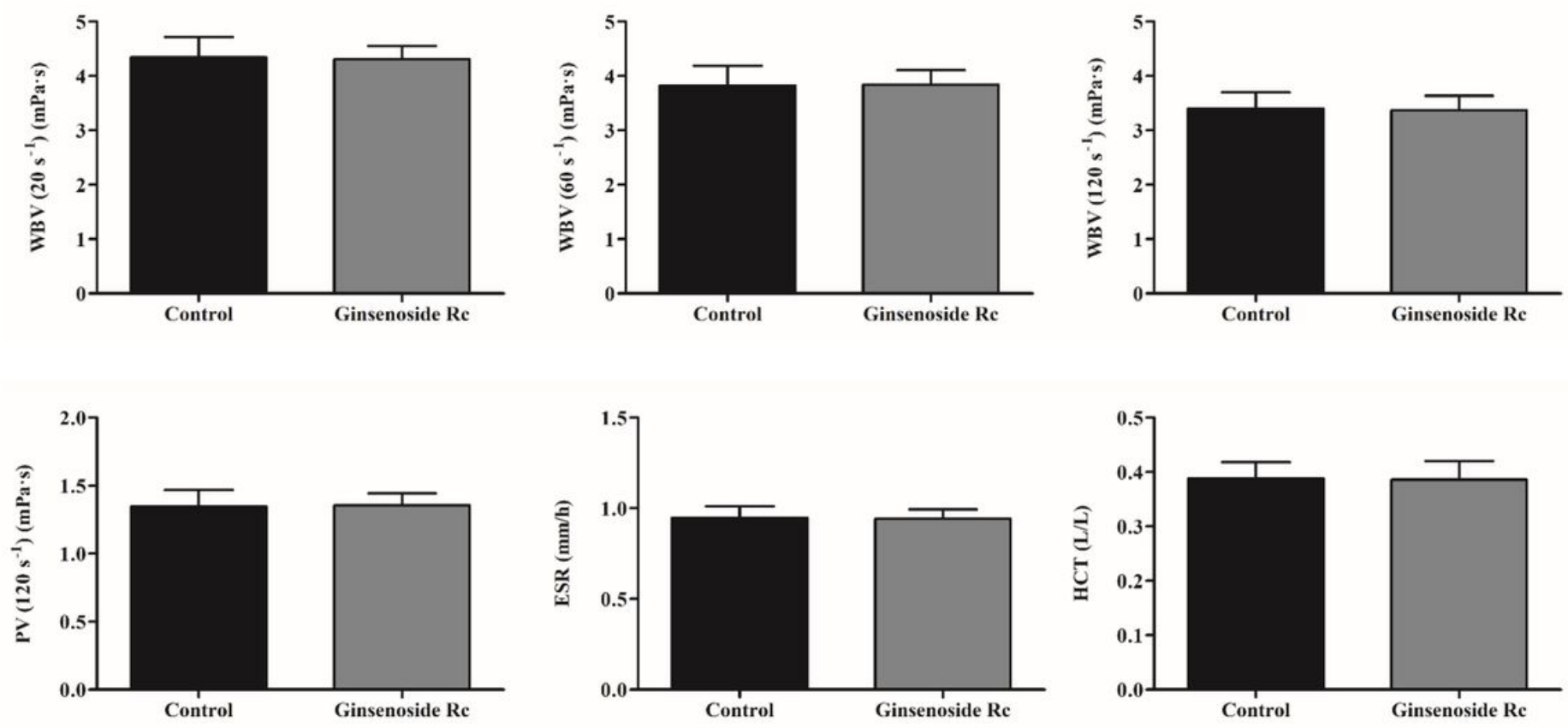

Figure 4

Effects of ginsenoside Rc on hemorheology in normal rats. Data were presented as the mean \pm SD. $n=10$ per group. Statistical analysis among various groups was conducted by one-way analysis of variance (ANOVA) with Tukey's post hoc test. 

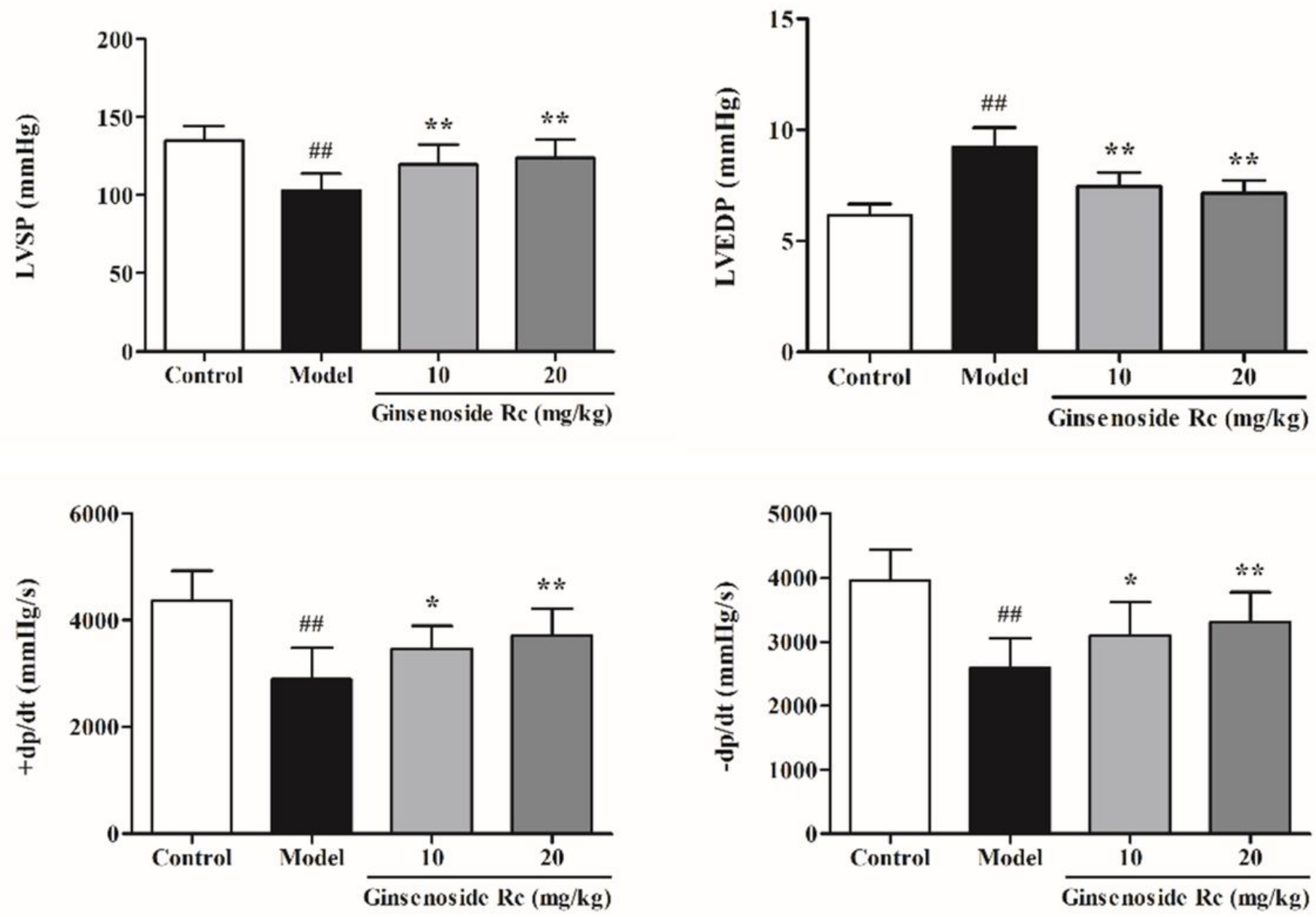

\section{Figure 5}

Effects of ginsenoside Rc on cardiac function in acute cold exposure rats. Data were presented as the mean \pm SD. $n=10$ per group. Statistical analysis among various groups was conducted by one-way analysis of variance (ANOVA) with Tukey's post hoc test. \#\#P<0.01, compared with the control group; ${ }^{*} \mathrm{P}<$ $0.05,{ }^{*} \mathrm{P}<0.01$, compared with the Model group.
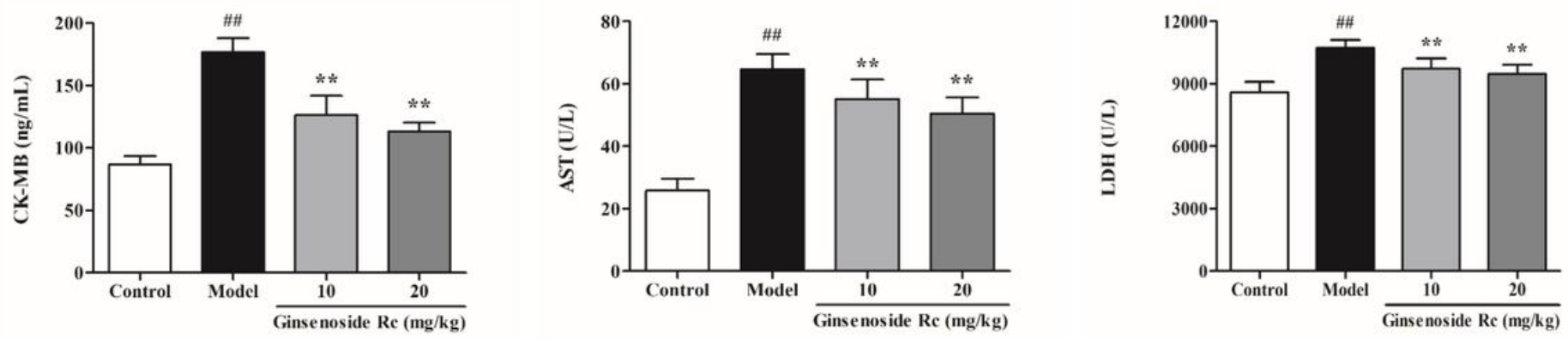

Figure 6

Effects of ginsenoside Rc on myocardial enzyme activities in acute cold exposure rats. Data were presented as the mean \pm SD. $n=10$ per group. Statistical analysis among various groups was conducted 
by one-way analysis of variance (ANOVA) with Tukey's post hoc test. \#\#P<0.01, compared with the control group; ${ }^{*} \mathrm{P}<0.01$, compared with the Model group.
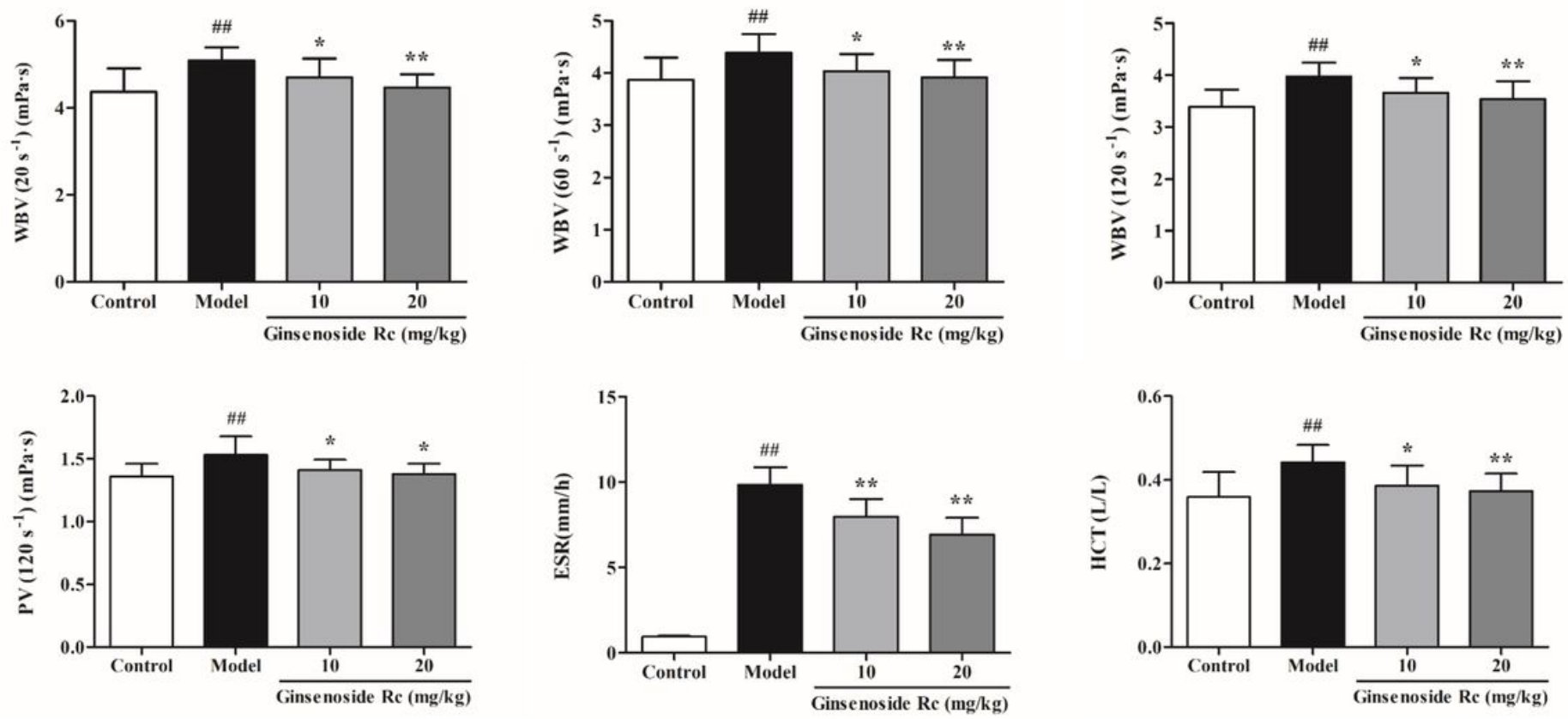

Figure 7

Effects of ginsenoside Rc on hemorheology in acute cold exposure rats. Data were presented as the mean \pm SD. $n=10$ per group. Statistical analysis among various groups was conducted by one-way analysis of variance (ANOVA) with Tukey's post hoc test. \#\#P<0.01, compared with the control group; ${ }^{*} \mathrm{P}<$ $0.05,{ }^{*} \mathrm{P}<0.01$, compared with the Model group.
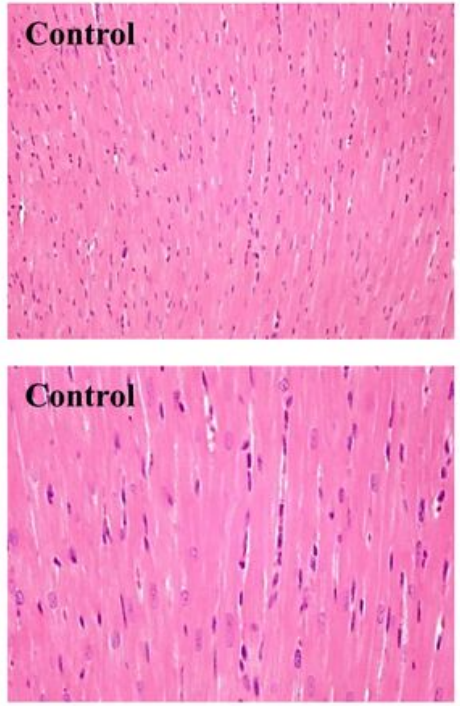
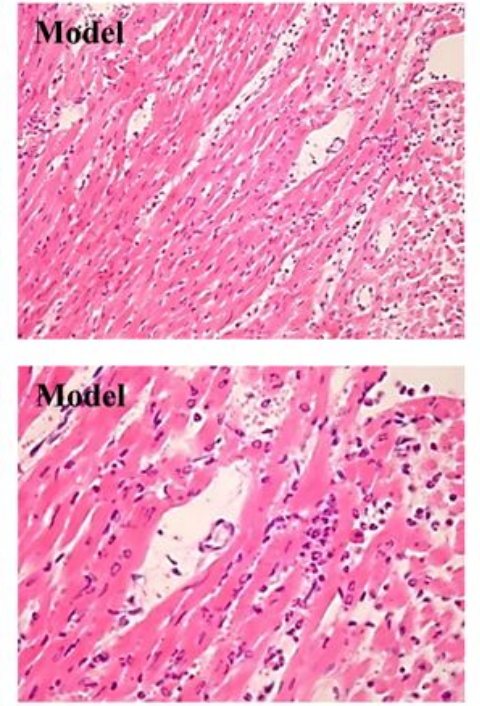
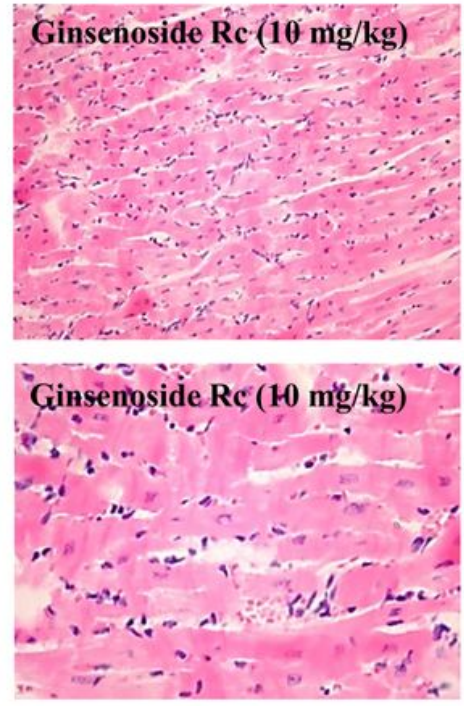
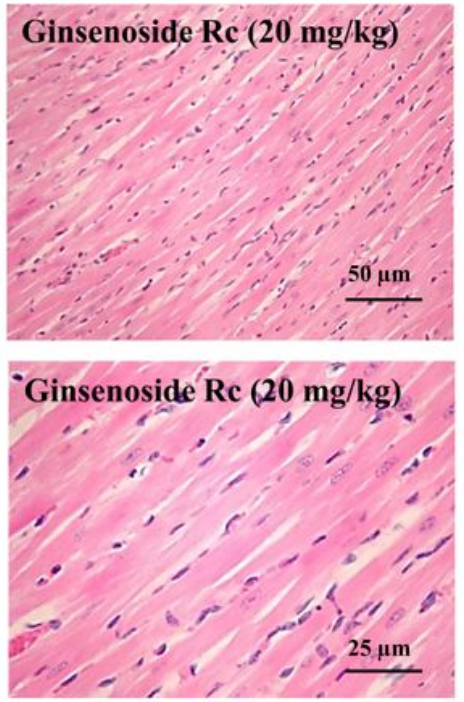

Figure 8 
Effects of ginsenoside Rc on histopathological examination of cardiac tissues in acute cold exposure rats. Representative hematoxylin-eosin (H\&E) pathological photomicrographs of left ventricular tissue. $\mathrm{n}=4$ per group. The cardiac apex was excised and fixed with $10 \%$ formaldehyde buffer for subsequent $\mathrm{H} \& \mathrm{E}$ staining. The sections were examined under light microscope, and then photomicrographs were taken. The top line: magnification $\times 200$; the bottom line: magnification $\times 400$.
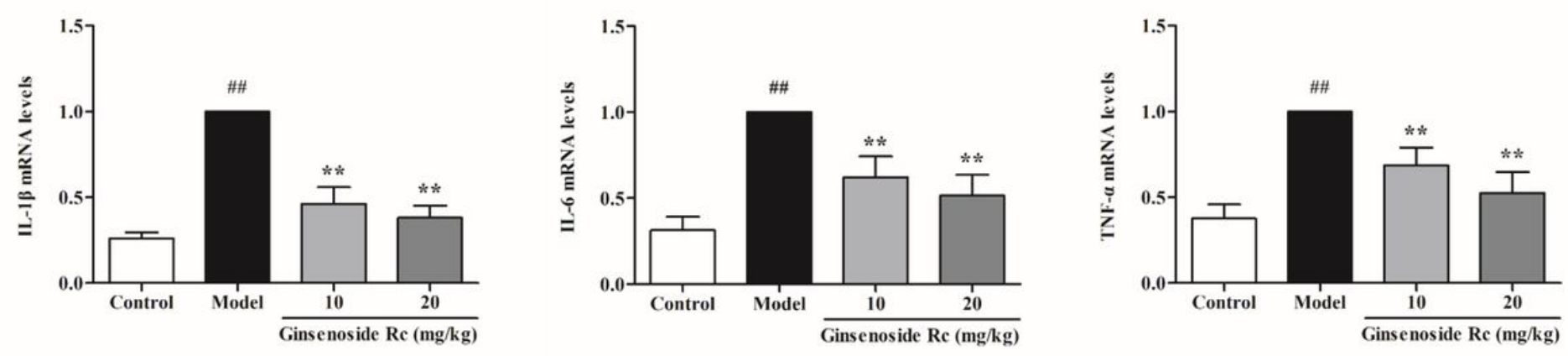

Figure 9

Effects of ginsenoside Rc on inflammation response in acute cold exposure rats. The mRNA expression levels of TNF- $a, \mathrm{IL}-1 \beta$ and IL- 6 in myocardium were measured. Data were presented as the mean \pm SD. $\mathrm{n}=10$ per group. Statistical analysis among various groups was conducted by one-way analysis of variance (ANOVA) with Tukey's post hoc test. \#\#P<0.01, compared with the control group; ${ }^{* * P}<0.01$, compared with the Model group.

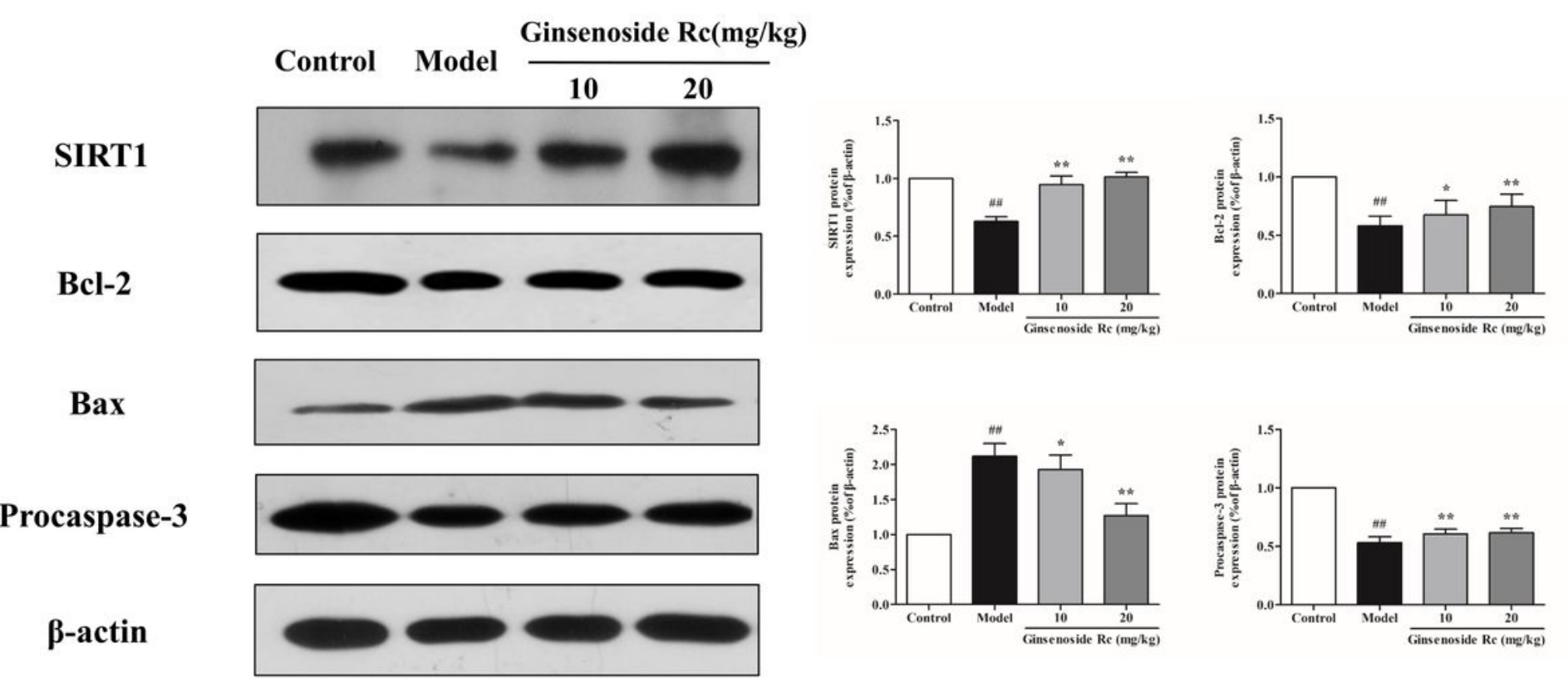

\section{Figure 10}

Effects of ginsenoside Rc on expression of SIRT1, Bcl-2, Bax and Procaspase-3 in acute cold exposure rats. Representative photograph and bar graph of SIRT1, Bcl-2, Bax and Procaspase-3. Data were presented as the mean \pm SD. $n=3$ per group. Statistical analysis among various groups was conducted by 
one-way analysis of variance (ANOVA) with Tukey's post hoc test. \#\#P< 0.01 , compared with the control group; ${ }^{*}<<0.05,{ }^{* *} \mathrm{P}<0.01$, compared with the Model group. 